\title{
Efficient Massive Multichannel Active Noise Control using Wave-Domain Adaptive Filtering
}

\author{
Sascha Spors and Herbert Buchner \\ Deutsche Telekom Laboratories, Technische Universität Berlin \\ Ernst-Reuter-Platz 7, 10587 Berlin, Germany \\ Email: \{sascha.spors,herbert.buchner\}@telekom.de
}

\begin{abstract}
Multichannel acoustic active noise control (ANC) systems are increasingly being developed in order to enlarge the spatial extend of the quiet zone. This paper discusses the physical background of an ideal ANC system for noise sources outside and within the ANC system. This idealized system can be realized reasonably well with a high number of channels (massive multichannel ANC). It is shown that the typically applied adaptation algorithms have fundamental limitations in the context of massive multichannel ANC. The versatile framework of eigenspace and wave-domain adaptive filtering is proposed as solution to these. Simulation of a massive multichannel ANC system illustrates the successful application of the proposed concepts.
\end{abstract}

\section{INTRODUCTION}

The active suppression of undesired noise by electroacoustical systems and digital signal processing has been an active field of research for several decades. Acoustic active noise control (ANC) is based on the principle of superposition using appropriately driven loudspeakers. In principle, a large zone where the noise is canceled (quiet zone) is desirable. Appropriate loudspeaker driving signals are typically generated by prefiltering a reference signal of the noise source. This reference signal is measured by appropriately placed microphones. The required pre-filters are derived by analyzing the residual error within the desired quiet zone. In order to cope with arbitrary noise fields and the time varying nature of acoustics, these prefilters are computed by adaptive algorithms. The size of the quiet zone heavily depends on the number of sensors and actuators used to analyze the wave fields and to create destructive interference. However, the underlying adaptation techniques show fundamental restrictions for high channel numbers. This paper discusses the physical basics of the acoustic problem, the restrictions of traditional adaptation algorithms and presents a novel adaptation scheme that overcomes these limitations. This scheme is based upon an explicit consideration of the wave nature of sound in the adaptation problem. The work presented in [1] is enhanced and extended by this contribution.

The following conventions are used throughout this paper: Vectors are denoted by lower case boldface, matrices by upper case boldface. The temporal frequency domain is denoted by underlining the quantities, the spatial transform domain (eigenspace, wave domain) by a tilde placed over the respective symbol. The two-dimensional position vector in Cartesian coordinates is given as $\mathbf{x}=\left[\begin{array}{ll}x & y\end{array}\right]^{T}$. The Cartesian coordinates are related to polar coordinates by $x=r \cos \alpha$ and $y=r \sin \alpha$. The discrete time index is denoted by $k$.

\section{ANC with ENLARged Quiet Zone}

An ANC system needs to perform a proper analysis of the reference and residual error field, and needs to provide suitable control over the wave field within the quiet zone. In order to understand the electroacoustical requirements for an ANC system with enlarged quiet zone, a closer look at the underlying physical principles is taken.

\section{A. Basic Concept}

The solution of the homogeneous wave equation in the (temporal) frequency domain for a bounded region $V$ with respect to inhomogeneous boundary conditions is given by the Kirchhoff-Helmholtz integral [2]

$$
\begin{aligned}
\underline{p}(\mathbf{x}, \omega)=-\oint_{\partial V}\left\{\underline{g}\left(\mathbf{x} \mid \mathbf{x}_{0}, \omega\right) \frac{\partial}{\partial \mathbf{n}} \underline{p}\left(\mathbf{x}_{0}, \omega\right)-\right. \\
\left.\quad-\underline{p}\left(\mathbf{x}_{0}, \omega\right) \frac{\partial}{\partial \mathbf{n}} \underline{g}\left(\mathbf{x} \mid \mathbf{x}_{0}, \omega\right)\right\} d S_{0},
\end{aligned}
$$

where $V$ denotes a bounded region surrounded by the border $\partial V, g\left(\mathbf{x} \mid \mathbf{x}_{0}, \omega\right)$ a suitably chosen Green's function, $p\left(\mathbf{x}_{0}, \omega\right)$ the acoustic pressure at the boundary $\partial V\left(\mathbf{x}_{0} \in \partial V\right)$ and $\frac{\partial}{\partial \mathbf{n}}$ the directional gradient in direction of the normal vector $\mathbf{n}$ of $V$. The Green's function has to fulfill the homogeneous boundary conditions imposed at $\partial V$. In the following, freefield Green's functions are of special interest. The formulation of the Kirchhoff-Helmholtz integral is independent from the dimensionality of the problem. The three-dimensional freefield Green's function can be interpreted as the field of a point source located at $\mathbf{x}_{0}$, the two-dimensional free-field Green's function as the field of a line source. A two-dimensional wave field is refereed here to as a field which is independent from one of the spatial coordinates. The free-field Green's function represents the wave field of a monopole source placed at the point $\mathbf{x}_{0}$, its directional gradient the field of a dipole source. Depending on the direction of the normal vector $\mathbf{n}$ two different types of problems are covered by the KirchhoffHelmholtz integral:

1) if $\mathbf{n}$ points inward an interior problem is considered, and

2) if $\mathbf{n}$ points outward an exterior problem.

We first discuss the interior problem and its implications for ANC.

For the interior problem $p(\mathbf{x}, \omega)$ denotes the pressure field inside $V(\mathbf{x} \in V)$. Note, that for this case $p(\mathbf{x}, \omega)$ equals zero 


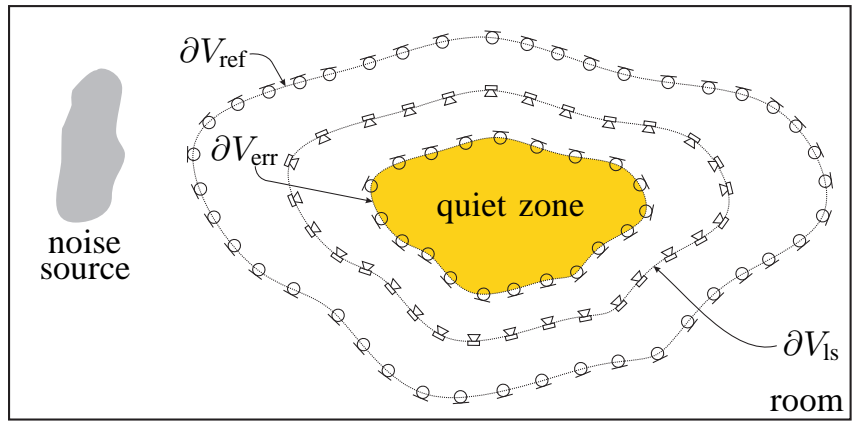

(a) Noise source located outside the system (exterior problem).

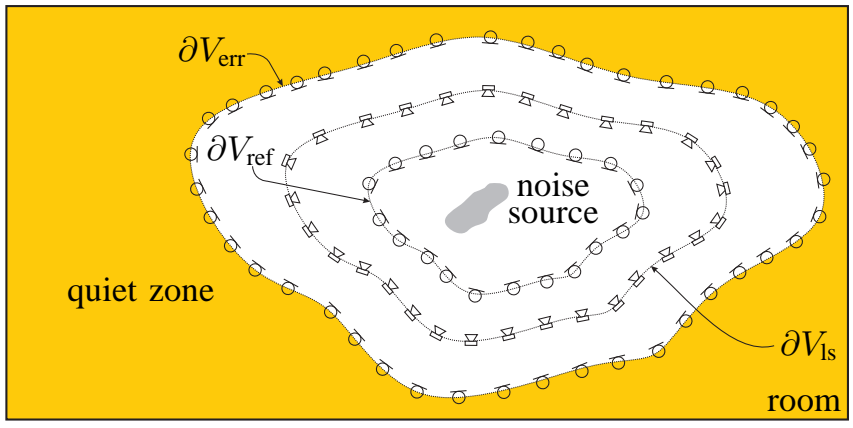

(b) Noise source located inside the system (interior problem).

Fig. 1. ANC setup with enlarged quiet zone (yellow shading) based on the Kirchhoff-Helmholtz integral.

outside $V$. Equation (1) can be interpreted in two ways: First, if the acoustic pressure and its directional gradient are known at the boundary $\partial V$ then the acoustic pressure inside $V$ can be calculated. Second, if a continuous distribution of monopole and dipole sources is placed on the border and driven properly, then the wave field in $V$ can be controlled fully. The first interpretation is the basis of wave field analysis (WFA) and extrapolation (WFE), while the second is the basis of many sound reproduction systems like, e.g., wave field synthesis (WFS). A suitable combination of both principles will result in an enlarged quiet zone for ANC.

Figure 1(a) illustrates such a combination. A distributed noise source located outside the ANC system emits noise that is scattered at the walls of a reverberant room. The resulting complex noise field and its residual (after compensation) is analyzed by reference microphones and error microphones arranged on the closed contours $\partial V_{\text {ref }}$ and $\partial V_{\text {err }}$. The wave field is controlled by loudspeakers arranged on the contour $\partial V_{\mathrm{ls}}$. For continuous microphone and loudspeaker distributions a perfect analysis and control of the wave field is possible according to Eq. (1). The result will be an enlarged quiet zone which fully covers $V_{\text {err }}$.

For the exterior problem the situation is depicted in Fig. 1(b): Here, the noise source is located within the ANC system and the resulting quiet zone is consequently outside the contour $\partial V_{\text {err }}$.

The next two subsections will introduce WFA and WFS as practical solutions for analysis and control of wave fields.

We limit ourselves to two-dimensional wave fields in the remainder of this contribution. An extension to the threedimensional case is straightforward.

\section{B. Wave Field Analysis}

It is convenient to represent acoustic wave fields with respect to an orthogonal basis for a wide variety of acoustic problems. The Kirchhoff-Helmholtz integral introduced in Section II-A states that it is possible to measure an arbitrary wave field on the boundary $\partial V$ of a bounded region $V$ to derive the wave field within that boundary. This principle combined with orthogonal wave field representations yields very efficient techniques for WFA as will be illustrated in the following.
The representations of a wave field that are connected to Cartesian and polar coordinates decompose a wave field into plane waves and circular harmonics, respectively [2]. Of special interest within the scope of this paper is the decomposition of an acoustic wave field into circular harmonics. This decomposition is given as follows

$$
\begin{aligned}
\underline{p}(\alpha, r, \omega)= & \sum_{\nu=-\infty}^{\infty} \underline{\breve{p}}^{(1)}(\nu, \omega) H_{\nu}^{(1)}\left(\frac{\omega}{c} r\right) e^{j \nu \alpha}+ \\
& \sum_{\nu=-\infty}^{\infty} \underline{\breve{p}}^{(2)}(\nu, \omega) H_{\nu}^{(2)}\left(\frac{\omega}{c} r\right) e^{j \nu \alpha},
\end{aligned}
$$

where $\underline{\breve{p}}^{(1),(2)}(\nu, \omega)$ denote the circular harmonics expansion coefficients, $H_{\nu}^{(1),(2)}$ the Hankel function of $\nu$-th order of first/second kind, $\nu$ the angular frequency and $c$ the speed of sound. It can be shown that $\underline{\breve{p}}^{(1)}(\nu, \omega)$ represents the expansion of an incoming wave field and $\breve{p}^{(2)}(\nu, \omega)$ the expansion of an outgoing wave field. The expansion coefficients in circular harmonics exhibit a direct link to the plane wave expansion coefficients

$$
\underline{\bar{p}}^{(1),(2)}(\theta, \omega)=\frac{4 \pi}{k} \sum_{\nu=-\infty}^{\infty} j^{\nu} \underline{\breve{p}}^{(1),(2)}(\nu, \omega) e^{j \nu \theta} .
$$

The plane wave expansion coefficients $\bar{p}^{(1)}(\theta, \omega)$ and $\underline{\bar{p}}^{(2)}(\theta, \omega)$ describe the spectrum of incoming/outgoing plane waves with incidence angle $\theta$.

It is possible to derive the circular harmonics decomposition coefficients very efficiently from microphone array measurements. Due to the underlying geometry of the circular harmonics, circular microphone arrays are well suited for this task. Suitable techniques can be found e.g. in [3]. The circular harmonics coefficients can be computed by transforming the microphone signals into the temporal and angular frequency domain and weighting them. One major benefit of circular microphone arrays is that their characteristics are independent from the incidence angle of the analyzed wave field.

In practice only a limited number of microphones will be used to measure the field on the circular boundary. This spatial sampling may lead to aliasing artifacts in the expansion coefficients. Due to the circular geometry no exact anti-aliasing 
condition can be given for arbitrary wave fields. However, it can be concluded that the amount of aliasing distortions depends on the number of angular measurement positions and the bandwidth of the measured field. For a given number of sampling positions a limitation of the bandwidth of the measured wave field limits spatial aliasing artifacts.

\section{Wave Field Synthesis}

WFS is a spatial sound reproduction technique whose theory is essentially based on the Kirchhoff-Helmholtz integral [4]. In the context of WFS, this fundamental principle can be interpreted as follows: A distribution of suitably driven monopole and dipole sources (secondary sources) placed on the boundary $\partial V$ allows the recreation of any desired virtual source wave field $\underline{s}(\mathbf{x}, \omega)$ within the entire listening area $V$.

Several simplifications of the Kirchhoff-Helmholtz integral are necessary to arrive at a realizable reproduction system. One of the key simplifications is to neglect the dipole secondary sources dictated by the Kirchhoff-Helmholtz integral (1). The reproduced wave field is then given as

$$
\underline{p}(\mathbf{x}, \omega)=-\oint_{\partial V} \underline{g}\left(\mathbf{x} \mid \mathbf{x}_{0}, \omega\right) \underbrace{2 a\left(\mathbf{x}_{\mathbf{0}}\right) \frac{\partial}{\partial \mathbf{n}} \underline{s}\left(\mathbf{x}_{0}, \omega\right)}_{\underline{d}\left(\mathbf{x}_{0}, \omega\right)} d S_{0},
$$

where $\underline{d}\left(\mathbf{x}_{0}, \omega\right)$ denotes the secondary source driving function and $a\left(\mathbf{x}_{\mathbf{0}}\right)$ a window function that takes care that only the relevant secondary sources are driven [5].

Typical systems aim at two-dimensional reproduction, more specifically at the reproduction of a spatial sound field in a listening area leveled with the listeners ears. WFS systems typically use loudspeakers with closed cabinets as secondary sources. These can be seen as approximation of point sources. However, for two-dimensional reproduction line sources would be the appropriate choice (see Section II-A). As a consequence of the applied approximations, several reproduction artifacts will be present that limit the control on the wave field within the listening area. Their influence on ANC and active listening room compensation have been investigated e. g. in [6], [7]. The most prominent artifact are amplitude errors.

In practice only a limited number of loudspeakers can be used. This spatial sampling may lead to aliasing artifacts in the reproduced wave field. In general, the amount of aliasing distortions will depend on the loudspeakers spacing, the geometry of the system and the bandwidth of the reproduced field. A detailed discussion of the aliasing artifacts of linear and circular WFS systems can be found e.g. in [8].

\section{Massive Multichannel ANC}

For a practical implementation of the proposed ANC schemes illustrated in Fig. 1(a) and Fig. 1(b), spatial sampling of the continuous microphone and loudspeaker distributions is required. The analysis and control capabilities of a particular ANC system depend amongst others on the amount of distortions by spatial aliasing. Hence, the size of the quiet zone and the bandwidth in which a suitable noise suppression can be gained depends on the number of microphones and

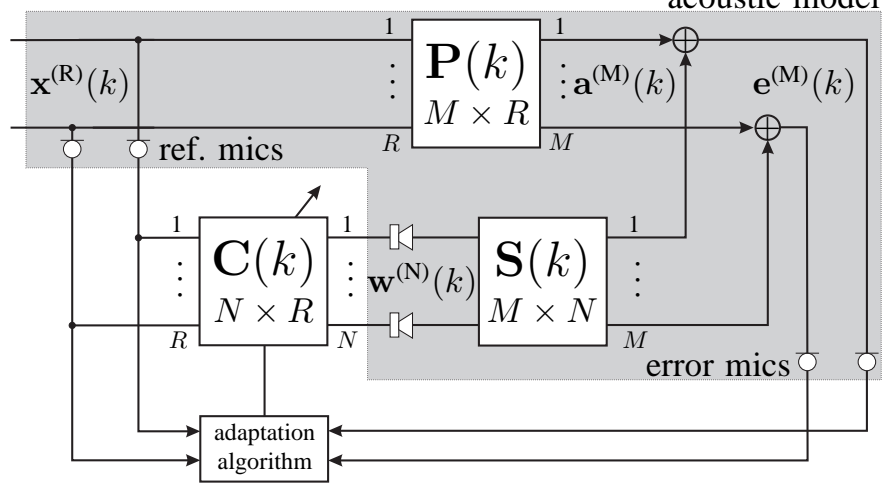

Fig. 2. Block diagram illustrating the generic feedforward broadband multichannel ANC system without feedback paths.

loudspeakers used.

Various ANC systems with multiple analysis and synthesis channels have been realized in the past [9]. However, the number of channels in multiple-input/multiple-output (MIMO) ANC systems was typically limited to less than ten channels. For an enhanced bandwidth and size of the quiet zone a high number of channels is required. The underlying signal processing techniques used for MIMO ANC systems show fundamental problems for high channel numbers. Therefore, we term systems with high channel numbers as massive multichannel systems in order to allow a distinction from traditional multichannel approaches.

\section{Traditional Adaptation Scheme Applied to Massive Multichannel ANC}

The following section illustrates the application of a traditional adaptation scheme to massive multichannel ANC and the resulting fundamental problems. Note, that both the interior and exterior problem are covered.

\section{A. Basic Structure}

The discrete time and space block diagram shown in Fig. 2 illustrates a generic multichannel ANC system without feedback paths. We assume that the effects of feedback from the loudspeakers to the reference microphones can be ignored, e.g. by use of proper acoustic damping. This is a typical assumption made for ANC systems. However, it is possible to account for the feedback paths also [9].

The matrices of impulse responses $\mathbf{P}(k), \mathbf{S}(k)$ and $\mathbf{C}(k)$ characterize the primary paths between the reference microphones and the error microphones, the secondary paths between the loudspeakers and the error microphones, and the compensation filters used to generate the loudspeaker signals from the reference signals. The elements of the matrices are composed from the finite impulse response from one input to one output at the $k$-th time instant. The signals of the reference microphones are combined in the vector $\mathbf{x}^{(\mathrm{R})}(k)=\left[x_{1}(k), x_{2}(k), \cdots, x_{R}(k)\right]^{T}$, where $x_{r}(k)$ denotes the signal of the $r$-th microphone. The signals from the error microphones are denoted by the vector $\mathbf{e}^{(\mathrm{M})}(k)$, the loudspeaker driving signals by $\mathbf{w}^{(\mathrm{N})}(k)$ and the noise field at the error microphones (without ANC) by $\mathbf{a}^{(\mathrm{M})}(k)$. 
The reference signal is filtered by the compensation filters and reproduced by the loudspeakers in order to archive the desired goal of a quiet zone. The primary path response $\mathbf{P}(k)$ and secondary path response $\mathbf{S}(k)$ are in general not known apriori and may change over time due to a varying acoustic environment. This calls for an adaptive computation of the compensation filters.

\section{B. Traditional Adaptation of Compensation Filters}

We briefly review the optimum least-squares solution for the adaptation problem introduced in Fig. 2. A detailed discussion can be found, e.g., in [10], [11]. The derived normal equation is the basis for various adaptation algorithms, like the filtered-x recursive least squares algorithm (X-RLS). The derived fundamental problems hold therefore for most of the currently applied multichannel adaptation algorithms.

The least-squares error cost function is given as

$$
\xi(\hat{\mathbf{c}}, k)=\sum_{\kappa=0}^{k} \lambda^{k-\kappa} \sum_{m=1}^{M}\left|e_{m}(\kappa)\right|^{2},
$$

where $0<\lambda<1$ denotes an exponential weighting factor. The normal equation is derived by expressing the error $\mathbf{e}^{(\mathrm{M})}(k)$ using the filter coefficients, introducing the result into the cost function (5) and calculating its gradient. The optimal filter coefficients in the mean-squared error sense are found by setting the gradient of the cost function to zero. The resulting normal equation is given as

$$
\hat{\boldsymbol{\Phi}}_{x x}(k) \hat{\mathbf{c}}(k)=\hat{\boldsymbol{\Phi}}_{x a}(k),
$$

where the $N_{c} N R \times 1$ vector $\hat{\mathbf{c}}(k)$ denotes a vector composed from all filter coefficients estimated at time instant $k$ and $N_{c}$ the number of filter coefficients. The $N R N_{c} \times N_{c} R N$ matrix $\hat{\boldsymbol{\Phi}}_{x x}$ denotes the averaged correlation matrix of the filtered reference signals. The filtered reference signals are defined by the convolution $x_{r}(k) * s_{m, n}(k)$ of the reference signals with the secondary path responses. The $N R N_{c} \times 1$ vector $\hat{\boldsymbol{\Phi}}_{x a}$ denotes the averaged correlation vector between these filtered reference signals and the noise field at the error sensors (without ANC). The optimal pre-equalization filter with respect to the cost function (5) is given by solving the normal equation (6). The X-RLS algorithm can be derived from the normal equation (6) by computing $\hat{\boldsymbol{\Phi}}_{x x}(k)$ and $\hat{\boldsymbol{\Phi}}_{x a}(k)$ in a recursive fashion and applying the matrix inversion lemma [10]. The calculation of the filtered reference signals requires knowledge of the secondary path response $\mathbf{S}(k)$, which is in general not known a-priori and may be time-variant. Hence, the secondary path characteristics have to be identified additionally in practice. This identification can be performed in two ways: off-line or on-line [9]. For the former techniques, the characteristics of the secondary path are measured using measurement signals before the ANC system is in operation. In the latter techniques, similar multichannel adaptation algorithms as outlined above are applied. These are simpler than the filtered-x techniques since they require no pre-filtering process. However, the same fundamental problems as outlined below for the pre-filter adaptation hold also for the identification of the secondary path [12]. Secondary path modeling errors will additionally influence the performance of the entire ANC system.

\section{Fundamental Problems of Traditional Filter Adaptation}

It can be concluded from the normal equation (6) that the adaptation of the compensation filters for the massive multichannel case is subject to fundamental problems:

1) Ill-conditioning of the correlation matrix $\hat{\boldsymbol{\Phi}}_{x x}(k)$, and

2) computational complexity for massive multichannel ANC systems.

Both problems are related to the solution of the normal Eq. (6) by the adaptive algorithm. The correlation matrix $\hat{\mathbf{\Phi}}_{x x}(k)$ expresses the auto- and cross-correlations of the filtered reference signals. Due to linear relations between the channels, and hence spatio-temporal correlations, $\hat{\boldsymbol{\Phi}}_{x x}(k)$ will generally be ill-conditioned. In the extreme case, depending on the reference signals $\mathbf{x}^{(\mathrm{R})}(k)$, there may be multiple possible solutions for $\hat{\mathbf{c}}$ that minimize $\xi(\hat{\mathbf{c}}, k)$.

Due to the dimensionality of the correlation matrix $\hat{\boldsymbol{\Phi}}_{x x}(k)$ the solution of the normal equation may become an infeasible task for massive multichannel ANC systems. The computational complexity of the filter adaptation is enormous even for modest channel numbers [10]. The same problems as outlined above hold also for the identification of the secondary path response.

\section{EFFICIENT AdAPTATION FOR MASSive MulTiCHANNEL ANC}

In order to overcome the fundamental problems of the traditional adaptation techniques in the context of massive multichannel ANC, the following section will propose an highly efficient adaptation scheme. It is based upon the concept of decoupling the MIMO systems $\underline{\mathbf{S}}(\omega)$ and $\underline{\mathbf{P}}(\omega)$ into a series of independent single channel systems by applying signal and system transformations. The optimal solution in the sense of decoupling is given by eigenspace adaptive filtering (EAF) that will be introduced in the next subsection.

The next steps require frequency-domain descriptions of the signals and systems introduced in Fig. 2. These are derived by transforming each element of the respective time-domain vectors and matrices with a discrete time Fourier transformation (DTFT).

\section{A. Eigenspace Adaptive Filtering}

This section gives a brief review of EAF, a detailed discussion can be found in [11]. The basic idea is to perform a decoupling of the MIMO systems $\underline{\mathbf{S}}(\omega)$ and $\underline{\mathbf{P}}(\omega)$ by applying a generalized singular value decomposition (GSVD). The decoupling of the MIMO systems represented by the matrices $\underline{\mathbf{S}}(\omega)$ and $\underline{\mathbf{P}}(\omega)$ is performed by pre-filtering them with their generalized singular matrix and post-filtering them with their respective right singular matrices. A decomposition of the compensation filter $\underline{\mathbf{C}}(\omega)$ is given in a similar way. Introducing the transformations into Fig. 2 yields Fig. 3 which illustrates the application of EAF to ANC. The necessary transformations are denoted by $\mathcal{T}_{1}, \mathcal{T}_{2}$ and $\mathcal{T}_{3}$. For EAF these 
acoustic model

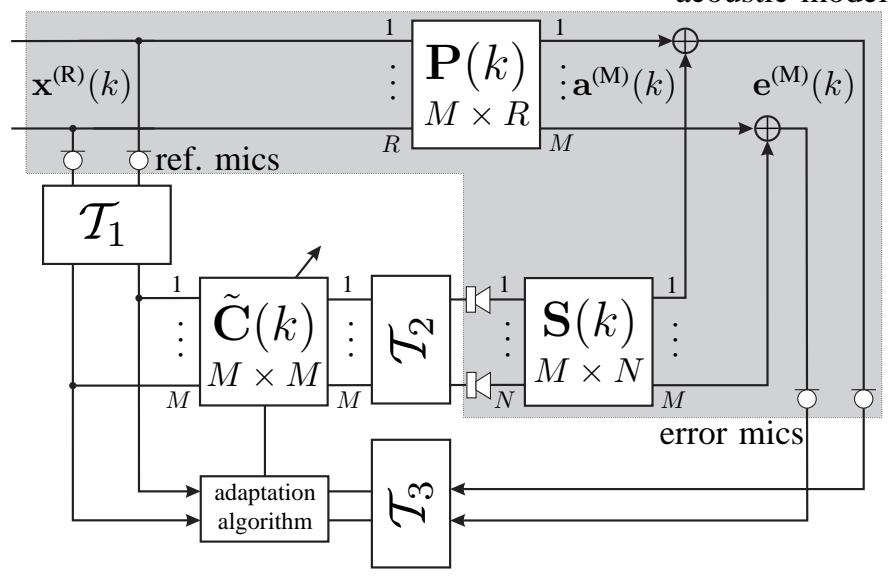

Fig. 3. Block diagram illustrating the eigenspace and wave-domain adaptive filtering approach to massive multichannel ANC.

transformations are given by MIMO systems represented by the singular matrices of $\underline{\mathbf{P}}(\omega)$ and $\underline{\mathbf{S}}(\omega)$.

It can be concluded from Fig. 3 that the adaptive inverse MIMO filtering problem is decomposed into $M$ single-channel adaptive inverse filtering problems using the GSVD. The adaptation of the compensation filters is performed independently for each of the transformed components. Note that the proposed transformations are independent from the particular adaptation algorithm used.

EAF provides a generic framework for MIMO pre-equalization which explicitly solves the second problem by utilizing signal and system transformations. The conditioning of $\hat{\boldsymbol{\Phi}}_{x x}(k)$ is also highly alleviated by removing all cross-channel correlations. Although EAF provides the optimal solution to massive multichannel ANC with respect to the desired decoupling, two major drawbacks remain: (1) the GSVD is computationally quite complex and (2) the optimal transformations depend on the secondary path response. The next section will introduce wave-domain adaptive filtering (WDAF) as practical solution to these shortcomings.

\section{B. Wave-Domain Adaptive Filtering using Circular Harmonics}

WDAF is based on approximation of the concept of perfect decoupling by explicit consideration of the characteristics of the propagation medium and the geometry of the underlying acoustic problem. The perfect decoupling of the MIMO adaptation problem is given up in favor of generic and fixed transformations $\mathcal{T}_{1}, \mathcal{T}_{2}$ and $\mathcal{T}_{3}$ (see Fig. 3) which are to some degree independent of the actual acoustic characteristics. Fixed transformations can provide nearly the same favorable properties as the optimal GSVD-based transformations with the benefit of computational efficiency.

It has been shown that the circular harmonics decomposition is a good candidate as wave-domain transformation for circular analysis geometries and not too reverberant acoustic environments [13]. In the following, the transformations $\mathcal{T}_{1}$, $\mathcal{T}_{2}$ and $\mathcal{T}_{3}$ are specialized for a wave-domain representation of the respective signals in circular harmonics decomposition

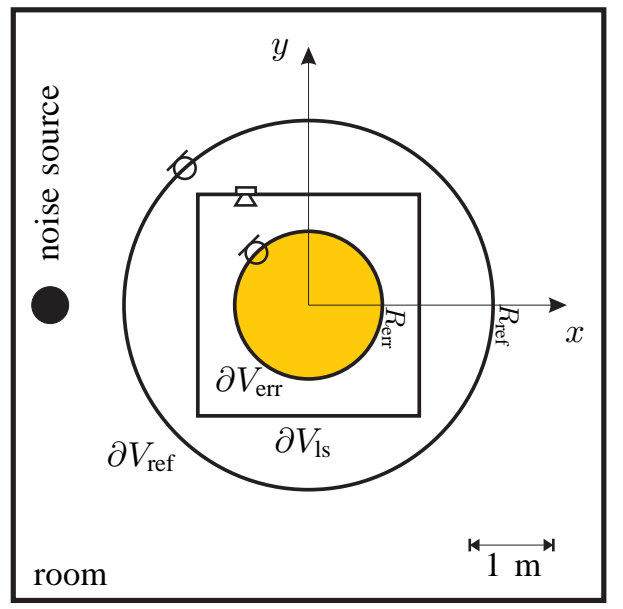

Fig. 4. Geometry of setup used for the simulated ANC system.

coefficients. Transformation $\mathcal{T}_{1}$ transforms the reference signal $\mathbf{x}^{(R)}(k)$ into its representation in terms of circular harmonics. If the reference signal is captured on a circular microphone array the techniques presented in [3] can be used for this purpose. Transformation $\mathcal{T}_{2}$ generates the loudspeaker driving signals from the filtered driving signals $\underline{\breve{\mathbf{w}}}^{(\mathrm{M})}$. Equation (2) together with a suitable loudspeaker selection criterion [5] can be used for this purpose. Transformation $\mathcal{T}_{3}$ calculates the circular harmonics decomposition coefficients of the residual error wave field within the quiet zone from the microphone array measurements. Again suitable techniques for circular microphone arrays are presented in [3].

The elegance and efficiency of WDAF lies in the combination of (1) decoupling the MIMO adaptation problem into a series of single channel problems by orthogonal wave field representations and (2) WFA by taking measurements only on the boundary of the quiet zone. Once the MIMO adaption problem is decoupled almost all known adaptation algorithms can be applied for the single channel adaptation problems.

The circular harmonics coefficients allow a discrimination between incoming and outgoing wave contributions. The incoming contributions of the reference and error signals are used for the exterior ANC problem (Fig. 1(a)), while for the interior ANC problem (Fig. 1(b)) the outgoing circular harmonics coefficients are used. Both adaptation problems are covered by Fig. 3 .

\section{Results}

The next section illustrates the application of WDAF to massive multichannel broadband feedforward ANC. For a proof of concept a numerically simulated environment was chosen.

\section{A. Simulation Setup}

Figure 4 illustrates the simulation setup. It consists of two circular microphone arrays for analysis of the reference and residual error field, and a loudspeaker array with rectangular geometry. The reference array has a radius of $R_{\text {ref }}=2.5 \mathrm{~m}$, 


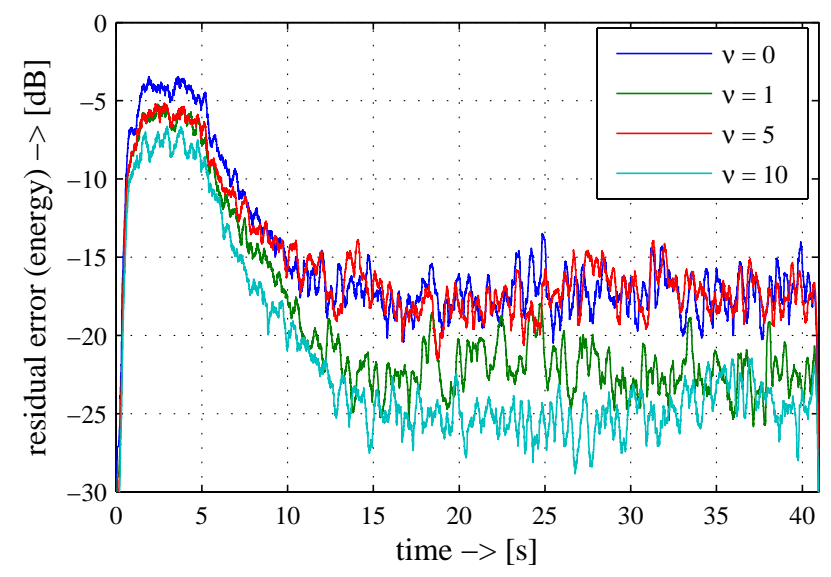

(a) Residual error signal for different circular harmonics.

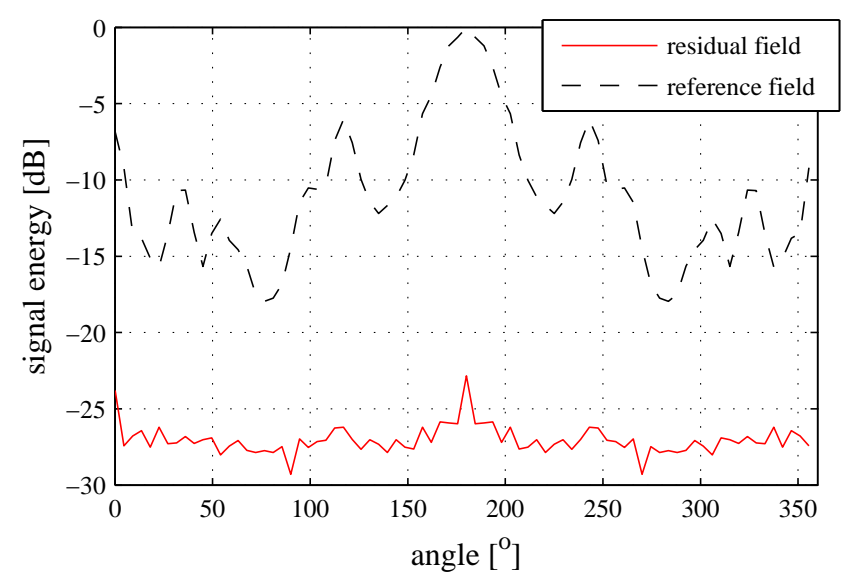

(b) Energy of plane wave components (after convergence).

Fig. 5. Results for simulated massive multichannel ANC system.

the error array of $R_{\text {err }}=1 \mathrm{~m}$. The loudspeaker array has a side length of $L_{\mathrm{ls}}=3 \mathrm{~m}$. All arrays have 80 spatial sampling points. The signals were bandlimited to a bandwidth of $800 \mathrm{~Hz}$ in order to avoid spatial aliasing. The simulation includes a reverberant room with size $8 \times 8 \mathrm{~m}(\mathrm{w} \times 1)$ and reflection factor $\rho_{\mathrm{pw}}=0.9$ at the side walls. The mirror image method was applied to simulate reflections. A noise source (point source) emitting white noise was placed at an angle of $\alpha=180^{\circ}$ and a distance of $D=3.50 \mathrm{~m}$ from the center of the arrays. For the adaptation, perfect knowledge of the secondary path was assumed and no noise was added to the microphone signals.

\section{B. Results}

Figure 5(a) illustrates the filter convergence by showing the energy of the error signal for different angular frequencies $\nu$. The case $\nu=0$ represents an omnidirectional pickup at the center of the quiet zone. It can be seen clearly that the adaptation of the compensation filters converges fast and stable for this massive multichannel scenario. A decomposition of the fields into plane waves allows insights into the spatial structure of the derived results. The plane wave decomposition has been derived from the circular harmonics coefficients by applying Eq. (3). Figure 5(b) shows the energy of the plane wave components of the reference and error field after convergence of the compensation filters. The suppression of the noise source over all incidence angles within the quiet zone can be seen clearly. This effectively results in an enlarged quiet zone.

\section{Conclusions}

This paper discusses the physical background of an ideal ANC system and its approximation by a massive multichannel system. Both interior and exterior noise control problems are discussed in a unified manner. It is shown that traditional adaptation schemes are subject to fundamental problems for massive multichannel systems. In order to overcome these, a highly efficient adaptation scheme is presented. It is based upon a decomposition of the MIMO adaptation problem into a series of single channel adaptation problems using physically motivated signal and system transformations. These transformations allow explicit consideration of the underlying physical problem in the adaptation algorithm. The simulation results presented show that WDAF provides fast and stable adaptation for massive multichannel ANC systems.

\section{REFERENCES}

[1] S. Spors and H. Buchner, "An approach to massive multichannel broadband feedforward active noise control using wave-domain adaptive filtering," in IEEE Workshop on Applications of Signal Processing to Audio and Acoustics, New Paltz, USA, October 2007.

[2] E. Williams, Fourier Acoustics: Sound Radiation and Nearfield Acoustical Holography. Academic Press, 1999.

[3] E. Hulsebos, D. de Vries, and E. Bourdillat, "Improved microphone array configurations for auralization of sound fields by Wave Field Synthesis," in 110th AES Convention. Amsterdam, Netherlands: Audio Engineering Society (AES), May 2001.

[4] A. Berkhout, "A holographic approach to acoustic control," Journal of the Audio Engineering Society, vol. 36, pp. 977-995, December 1988.

[5] S. Spors, "Extension of an analytic secondary source selection criterion for wave field synthesis," in 123th AES Convention. New York, USA: Audio Engineering Society (AES), October 2007.

[6] A. Kuntz and R. Rabenstein, "An approach to global noise control by wave field synthesis," in European Signal Processing Conference (EUSIPCO), Vienna, Austria, Sept. 2004.

[7] S. Spors, M. Renk, and R. Rabenstein, "Limiting effects of active room compensation using wave field synthesis," in 118th AES Convention. Barcelona, Spain: Audio Engineering Society (AES), May 2005.

[8] S. Spors and R. Rabenstein, "Spatial aliasing artifacts produced by linear and circular loudspeaker arrays used for wave field synthesis," in 120th AES Convention. Paris, France: Audio Engineering Society (AES), May 2006.

[9] S. Kuo and D. Morgan, Active Noise Control Systems. Algorithms and DSP Implementations. Wiley, June 1996.

[10] M. Bouchard and S. Quednau, "Multichannel recursive-least-squares algorithms and fast-transversal-filter algorithms for active noise control and sound reproduction systems," IEEE Transactions on Speech and Audio Processing, vol. 8, no. 5, pp. 606-618, September 2000.

[11] S. Spors, H. Buchner, and R. Rabenstein, "Eigenspace adaptive filtering for efficient pre-equalization of acoustic MIMO systems," in European Signal Processing Conference (EUSIPCO), Florence, Italy, September 2006.

[12] H. Buchner, S. Spors, and W. Kellermann, "Wave-domain adaptive filtering: Acoustic echo cancellation for full-duplex systems based on wave-field synthesis," in IEEE International Conference on Acoustics, Speech, and Signal Processing (ICASSP), Montreal, Canada, 2004.

[13] S. Spors and R. Rabenstein, "Evaluation of the circular harmonics decomposition for WDAF-based active listening room compensation," in 28th AES Conference: The Future of Audio Technology - Surround and Beyond. Pitea, Sweden: Audio Engineering Society (AES), June 2006, pp. 134-149. 